Schwingungsspektroskopische Untersuchungen anorganischer Festkörper XIV ${ }^{1}$

\title{
IR-Spektren kristalliner, wasserfreier Permanganate, Pertechnetate und Perrhenate
}

\author{
Achim Müller und Bernt Krebs* \\ Anorganisch-Chemisches Institut der Universität Göttingen \\ (Z. Naturforschg. 21 b, 3-7 [1966] ; eingegangen am 7. Oktober 1965)
}

\begin{abstract}
The infrared absorption frequencies in the region of the $\mathrm{MeO}_{4}{ }^{\ominus}$ fundamental vibrations are reported for several crystalline permanganates, pertechnetates, and perrhenates. On the basis of the IR spectra of the crystals and of additional considerations correct frequencies are assigned to the $v_{2}$ (E) vibrations of the $\mathrm{MeO}_{4}{ }^{\ominus}$ tetrahedra. The influence of the crystal field on the fundamental vibrations of the $\mathrm{MnO}_{4}{ }^{\ominus}, \mathrm{TcO}_{4}{ }^{\ominus}$, and $\mathrm{ReO}_{4}{ }^{\ominus}$ ions is discussed, using site group analysis and factor group considerations.
\end{abstract}

Während für Salze der Hauptgruppenelemente eingehende und vergleichende Untersuchungen über den Einfluß des Kristallfeldes und der Art des Kations auf die inneren Schwingungen von Anionenpolyedern im Gitter vorliegen ${ }^{2}$, fehlen bisher entsprechende Arbeiten über Salze der Übergangsmetalle ${ }^{3}$.

Um vor allem die Anwendbarkeit Gruppen-theoretischer Methoden auf die Spektren von Salzen der Nebengruppenelemente im Vergleich zu entsprechenden Salzen der Hauptgruppenelemente zu prüfen, haben wir IR-Spektren von kristallinen, wasserfreien Permanganaten, Pertechnetaten und Perrhenaten im Bereich der inneren Schwingungen gemessen.

Die Grundschwingungen von $\mathrm{MnO}_{4}{ }^{\ominus}$, $\mathrm{TcO}_{4}{ }^{\ominus}$ und $\mathrm{ReO}_{4}{ }^{\ominus}$

Für die freien Ionen mit der Symmetrie $T_{d}$ klassifizieren sich die eigentlichen Normalschwingungen nach den irreduziblen Darstellungen ${ }^{4}$

$$
\Gamma_{\text {schw. }}=\mathrm{A}_{1}(\mathrm{R})+\mathrm{E}(\mathrm{R})+2 \mathrm{~F}_{2}(\mathrm{R}, \mathrm{IR}) .
$$

1 Zugleich VI. Mitteilung über Rhenium-Sauerstoff-Verbindungen; XIII. bzw. V. Mitt.: A. Müller, Z. Naturforschg. 21 a, im Druck.

* Zur Zeit: Brookhaven National Laboratory, Upton, New York, USA.

2 Vgl. u. a. E. Steger u. W. Schmidt, Ber. Bunsenges. physik. Chem. 68, 102 [1964]. (Sulfate und Phosphate.)

3 Spektren zahlreicher Oxosalze von Übergangsmetallen werden ohne Diskussion von F. A. Miller u. C. H. W IL- $_{\text {- }}$ kins, Analyt. Chem. 24, 1253 [1952] u. F. A. Miller, G. L. Carlson, F. F. Bentley u. W. H. Jones, Spectrochim. Acta [London] 16, 135 [1960] angegeben.

4 Nachweis tetraedrischer Struktur für $\mathrm{ReO}_{4} \odot$ in wäßriger Lösung: a) H. H. Claassen u. A. J. Zielen, J. chem. Physics 22, 707 [1954] ; b) L. A. Woodward u. H. L. Roberts, Trans. Faraday Soc. 52, 615 [1956] ; c) für $\mathrm{TcO}_{4}{ }^{\ominus}: \mathrm{R}$. H. Busey u. O. L. Keller, J. chem. Physics 41, 215 [1964].
Für das $\mathrm{MnO}_{4}{ }^{\ominus}$-Ion in Lösung liegt bisher kein vollständiges Schwingungsspektrum vor. Es sind lediglich verschiedene kristalline Permanganate IR-spektroskopisch untersucht worden ${ }^{3,5,6}$. Bisher versuchte Zuordnungen ${ }^{5,6}$ sind unvollkommen, da die $v_{2}(\mathrm{E})$ entsprechende Bande wegen zu geringer Intensität nicht beobachtet werden konnte, obwohl sie nach den Auswahlregeln der Site-Symmetrie für fast alle bisher spektroskopisch untersuchten Permanganate erlaubt ist (für $T_{d}$-Symmetrie jedoch verboten). Weiterhin sind Zuordnungen z. T. unrichtig ${ }^{5}$ und in Tabellen nicht richtig übernommen worden ${ }^{7}$. Eine bis auf $\nu_{2}$ (nicht gemessen) richtige Zuordnung für $\mathrm{KMnO}_{4}$ wird von STEGer und DANzer ${ }^{8}$ angegeben.

Die Grundschwingungen des $\mathrm{MnO}_{4}{ }^{\ominus}$-Ions gehen aus Tab. 1 hervor. Die Frequenzen sind Mittelwerte verschiedener Alkalipermanganate. Die Frequenz für $v_{2}$ ist nicht absolut sicher; sie wurde von uns durch Abschätzung mit Hilfe der Beziehung ${ }^{9}$

$$
\frac{v_{3} \cdot v_{4}}{v_{1} \cdot v_{2}}=\sqrt{\frac{2}{3}\left(1+\frac{4 m_{0}}{m_{\mathrm{Me}}}\right)}
$$

5 C. Rocchiccioli, C. R. hebd. Séances Acad. Sci. 256, 1707 [1963] ; C. J. Ballhausen, Theor. Chim. Acta 1, 285 [1963] (Zuordnung der von Miller ${ }^{3}$ et al. gemessenen Schwingungsfrequenzen).

6 A. Müller u. B. Krebs, Naturwissenschaften 52, 448 [1965].

7 K. Naкамото, Infrared Spectra of Inorganic and Coordination Compounds, Wiley \& Sons, New York und London 1963; Druckfehler auf S. 107: für $\mathrm{MnO}_{4}{ }^{\ominus}$ muß es für $v_{4}\left(\mathrm{~F}_{2}\right)$ statt $500400 \mathrm{~cm}^{-1}$ heißen, entsprechend: H. SIEBERT, Z. anorg. allg. Chem. 275, 225 [1954].

8 E. Steger u. K. Danzer, Ber. Bunsenges. physik. Chem. 68, 635 [1964].

9 Diese Beziehung ergibt sich aus einer einfachen Valenzkraftmodell-Rechnung; vgl. G. Herzberg, Infrared and R a m a n Spectra of Polyatomic Molecules, Van Nostrand, New York 1945. 
und an Hand des Spektrums von $\mathrm{Ba}\left(\mathrm{MnO}_{4}\right)_{2}$ wahrscheinlich gemacht ${ }^{10}$. Das Verhältnis $v_{4} / v_{2}>1$ ist vernünftig, wie ein Vergleich mit den Spektren anderer tetraedrischer Oxoanionen zeigt ${ }^{11}$.

\begin{tabular}{|c|c|c|c|c|c|}
\hline & $v_{1}$ & \multicolumn{1}{|c|}{$v_{2}$} & $v_{3}$ & $v_{4}$ & Lit. \\
\hline $\mathrm{MnO}_{4} \ominus$ & 845 & $(355) *$ & 910 & 395 & $* *$ \\
$\mathrm{TcO}_{4} \ominus$ & 912 & $(347) * * *$ & 912 & 325 & $4 \mathrm{c}$ \\
$\mathrm{ReO}_{4} \ominus$ & 971 & $(350) * * *$ & 918 & 331 & $4 \mathrm{a}$ \\
\hline
\end{tabular}

Tab. 1. Grundschwingungen der Ionen $\mathrm{MnO}_{4}{ }^{\ominus}, \mathrm{TcO}_{4}{ }^{\ominus}$ und $\mathrm{ReO}_{4} \odot$ (Frequenzen in $\mathrm{cm}^{-1}$ ). * Frequenz wurde aus einer Rechnung und aus dem IR-Spektrum von $\mathrm{Ba}\left(\mathrm{MnO}_{4}\right)_{2}$ wahrscheinlich gemacht. ${ }^{* *}$ Angegebene Frequenzen stellen Mittelwerte aus IR-Spektren verschiedener Alkalipermanganate dar (vgl. Tab. 3). *** Mittelwerte aus verschiedenen Kristallspektren; in Lösung nicht zu beobachten.

Kürzlich haben Busey und Keller ${ }^{4 c}$ über das $\mathrm{R}$ a m a n - Spektrum des $\mathrm{TcO}_{4}{ }^{\ominus}$-Ions in wäßriger Lösung berichtet. Nach Messungen der Autoren sind $v_{1}\left(\mathrm{~A}_{1}\right)$ und $v_{3}\left(\mathrm{~F}_{2}\right)$ zufällig entartet. Ebenfalls wegen (näherungsweise) zufälliger Entartung liegt die $v_{2}$ entsprechende $\mathrm{R}$ a $\mathrm{m}$ a $\mathrm{n}$ - Linie dicht neben der intensiveren $v_{4}\left(\mathrm{~F}_{2}\right)$-Linie und konnte nicht beobachtet werden. Inzwischen wurde von MüLLER und KREBS ${ }^{12}$ die Bande der Schwingung $v_{2}(\mathrm{E})$ im IR-Festkörperspektrum von $\mathrm{NH}_{4} \mathrm{TcO}_{4}$ und $\mathrm{TlTcO}_{4}$ ermittelt (in Tab. 1 in Klammern angegeben).

Ebenso konnte für das $\mathrm{ReO}_{4}{ }^{\ominus}$-Ion in wäßriger Lösung durch Raman-spektroskopische Untersuchungen von ClaAssen und Zielen ${ }^{4 a}$ einerseits und Woodward und Roberts ${ }^{4 \mathrm{~b}}$ andererseits $v_{2}(\mathrm{E})$ nicht ermittelt werden. Eine frühere Zuordnung des $\mathrm{R}$ a m a n - Spektrums von $\mathrm{KReO}_{4}$ und $\mathrm{NaReO}_{4}$ von Fonteyne ${ }^{13}$ ist nicht richtig. Fonteyne trifft eine $\mathrm{Zu}$ ordnung $v_{2}(\mathrm{E})>v_{4}\left(\mathrm{~F}_{2}\right)$. Ebenso können wir eine von Duval und Lecomte ${ }^{14}$ getroffene Zuordnung einer beim $\mathrm{KReO}_{4}$ IR-spektroskopisch ermittelten Bande bei $364 \mathrm{~cm}^{-1}$ zu $v_{4}\left(\mathrm{~F}_{2}\right)$ nicht bestätigen. Von den Autoren wird keine weitere Bande im Bereich

10 Es ist allerdings auch möglich, daß $v_{2}$ mit $v_{4}$ zusammenfällt, da in fast allen Spektren der Permanganate eindeutig keine Bande auftritt, die $v_{2}$ zuzuordnen wäre.

11 Zur Diskussion des Frequenzverhältnisses $v_{4}\left(\mathrm{~F}_{2}\right) / v_{2}(\mathrm{E})$ bei verschiedenen Oxosalzen der Übergangsmetalle vgl. l. c. ${ }^{12,15}$ sowie A. Müller und B. Krebs, Z. anorg. allg. Chem., im Druck.

12 A. Müller u. B. Krebs, Z. Naturforschg. 20 a, 967 [1965].

13 R. Fonteyne, Natuurwetensch. Tijdschr. 20, 20 [1938]; vgl. K. W. F. Kohlrausch, R a m a n - Spektren, Akademische Verlagsgesellschaft, Leipzig 1943.

14 C. Duval u. J. Lecomte, C. R. Hebd. Séances Acad. Sci. 239, 249 [1954]; vgl. J. Leсомте, Spectroscopie dans l'infrarouge, in: Handbuch der Physik, Band XXVI, S. $825,1958$. $\operatorname{der} \delta(\mathrm{ORe} 0)$-Schwingungen angegeben. Unsere neueren Messungen an $\mathrm{KReO}_{4}$ hatten ergeben, daß die Bande bei $361 \mathrm{~cm}^{-1}$ im IR-Spektrum $v_{2}(\mathrm{E})$ zugeordnet werden muß ${ }^{15}$.

$$
\begin{aligned}
& \text { Zur Theorieder Festkörper- } \\
& \text { Schwingungspektren }{ }^{16}
\end{aligned}
$$

Ein Kristall, der aus $N$ Einheitszellen besteht, wobei jede Einheitszelle $n$ Atome enthält, hat $3 n N$ Schwingungsfreiheitsgrade ${ }^{*}$ und damit $3 n N$ Normalschwingungen ${ }^{*}$. Von diesen $3 n N$ Normalschwingungen können jedoch lediglich $3 n-3$ als Grundschwingungen (optische Schwingungen) im IR- und im R a m a n-Spektrum auftreten. Die optischen Schwingungen eines Kristalls entsprechen Schwingungen, bei denen äquivalente Gitterpunkte in Phase schwingen.

Die optischen Schwingungen lassen sich nach den irreduziblen Darstellungen der Einheitszellengruppe oder Faktorgruppe klassifizieren, die als Elemente die Translationsgruppe als invariante Untergruppe der Raumgruppe und deren Nebengruppen enthält und die zu einer Punktgruppe isomorph ist. Dabei lassen sich die inneren Schwingungen (innerhalb der Ionen- bzw. Molekülgruppierungen) von den äußeren Schwingungen („Gitterschwingungen“) getrennt behandeln.

Neben der von Bhagavantam und VenkatarayUdu ${ }^{17}$ entwickelten Methode der Klassifizierung der Normalschwingungen nach den irreduziblen Darstellungen der Faktorgruppe lassen sich nach HaLFORD ${ }^{18}$ in Kristallen mit Molekülen oder Ionengruppierungen der inneren Schwingungen nach der sogenannten Site-Gruppe klassifizieren. Die Site-Gruppe ist einerseits zu einer Untergruppe der Einheitszellengruppe isomorph und zum anderen gleich einer Untergruppe der Punktgruppe des freien Moleküls bzw. (Komplex-) Ions. Hornig ${ }^{19}$ konnte weiterhin

15 A. Müller, Z. Naturforschg. 20 a, 745 [1965].

16 Vgl. u. a.: a) F. Matossi, Gruppentheorie der Eigenschwingungen von Punktsystemen, Springer-Verlag, Berlin 1961; b) S. Bhagavantam u. T. Venkatarayudu, Theory of Groups and its Application to Physical Problems, Waltair [Indien] 1962 ; c) S. S. Mitra u. P. J. Gielisse, in: Progress in Infrared Spectroscopy, Vol. II, Plenum Press, New York 1964 ; d) H. Winston u. R. S. Halford, J. chem. Physics 17, 607 [1949].

* Einschließlich der uneigentlichen Schwingungen.

17 S. Bhagavantam u. T. Venkatarayudu, Proc. Indian Acad. Sci. Sect. A 9, 224 [1939]; S. Bhagavantam, Proc. Indian Acad. Sci. Sect. A 13, 513 [1941].

18 R. S. Halford, J. chem. Physics 14, 8 [1946].

19 D. F. Hornig, J. chem. Physics 16, 1063 [1948]. 
zeigen, daß man mit beiden Methoden äquivalente Resultate erhalten kann.

Die inneren Schwingungen der hier untersuchten Oxoanionen lassen sich in O-Näherung mit einem Modell mit Ionen als orientiertes Gas im Gitter ohne Wechselwirkung (mit der Symmetrie des freien Ions) behandeln. In 1. Näherung kann die Wirkung der Nachbarionen auf ein bestimmtes Ion als effektives Störfeld mit bestimmter Symmetrie (statisches Feld) berücksichtigt werden, wodurch die ursprüngliche Symmetrie zur Site-Symmetrie erniedrigt wird. Berücksichtigung intermolekularer bzw. interionischer Wechselwirkungen in der Elementarzelle (2. Näherung; dynamisches Feld) entspricht einer oben erwähnten Faktorgruppenanalyse.

Diskussion der inneren Schwingungen in kristallinen Permanganaten, Per. technetaten und Perrhenaten

Wie weit machen sich die Auswahlregeln der Siteoder Faktorgruppe in den Spektren der untersuchten Verbindungen bemerkbar?

In Tab. 2 sind die Auswahlregeln für die verschiedenen Site-Gruppen und Faktorgruppen zusammengestellt; aus Tab. 3 gehen die ermittelten Schwingungsfrequenzen hervor. Sehr schwache Banden, die z. T. nur bei größeren Probenkonzentrationen zu erkennen waren und die sich eindeutig Oberschwingungen (der inneren Schwingungen) oder Kombinationsschwingungen (zwischen inneren einerseits und inneren und äußeren andererseits) zuordnen ließen, wurden nicht mit angegeben, da sie für eine Diskussion im Rahmen dieser Arbeit nicht wichtig sind (vgl. l. c. ${ }^{4 \mathrm{c}}$ ). Hierzu gehören u. a. die von uns in einer früheren Arbeit ${ }^{15}$ angegebenen Schwingungsfrequenzen $866 \mathrm{~cm}^{-1}$ von $\mathrm{KReO}_{4}$ und $\mathrm{NH}_{4} \mathrm{ReO}_{4}{ }^{15}$. Weiterhin sind die den inneren Schwingungen des $\mathrm{NH}_{4}{ }^{\oplus}$-Ions entsprechenden Frequenzen in Tab. 3 nicht mit ausgeführt. Da die von uns ${ }^{15}$ und Busey und Keller ${ }^{4 c}$ in einer früheren Arbeit angegebene Aufspaltung der Bande mit einem Zentrum bei $910 \mathrm{~cm}^{-1}$ (Tab. 3) vom $\mathrm{KReO}_{4}$ und $\mathrm{NH}_{4} \mathrm{ReO}_{4}$ nicht deutlich und nicht immer reproduzierbar ist, muß als wahrscheinlich angenommen werden, daß die Frequenzen 929 und $910 \mathrm{KReO}_{4}$ bzw. 930 und $910\left(\mathrm{NH}_{4} \mathrm{ReO}_{4}\right)$ den beiden SiteGruppen-Grundschwingungen zur irreduziblen Darstellung $\mathrm{B}$ und $\mathrm{E}$ von $\mathrm{C}_{4 \mathrm{~h}}$ entsprechen (vgl. Tab. 3).

Ein Vergleich der Anzahl der nach der Faktorgruppe möglichen Schwingungen mit der Anzahl der gemessenen zeigt, daß eine durch Kopplung der inneren Schwingungen der $\mathrm{MeO}_{4}{ }^{\ominus}$-Einheiten in der Elementarzelle mögliche Korrelationsfeld-Aufspaltung praktisch nicht beobachtet wird. Lediglich beim $\mathrm{Ba}\left(\mathrm{MnO}_{4}\right)_{2}$ sind Anzeichen für eine Korrelationsfeld-Aufspaltung von $v_{3}\left(\mathrm{~F}_{2}\right)$ vorhanden. Es ist allerdings zu bemerken, daß bei der Faktorgruppe $\mathrm{C}_{4 \mathrm{~h}}$ (Substanzen, die in der Raumgruppe $\mathrm{C}_{4 \mathrm{~h}}^{6}$ kristallisieren) eine Kopplung zu keiner Erhöhung der Zahl der IR-aktiven Schwingungen ge-

\begin{tabular}{|c|c|c|c|c|c|c|c|}
\hline \multirow{2}{*}{$\begin{array}{l}\text { Punkt- } \\
\text { gruppe des } \\
\text { freien Ions }\end{array}$} & \multicolumn{2}{|c|}{$\mathrm{C}_{4 \mathrm{~h}}^{6} Z / 2=2$} & \multicolumn{2}{|c|}{$\mathrm{D}_{2 \mathrm{~h}}^{16} Z=4$} & \multicolumn{2}{|c|}{$\mathrm{C}_{2 \mathrm{~h}}^{5} Z=4$} & \multirow{2}{*}{$\frac{\mathrm{D}_{2 \mathrm{~h}}^{24} Z=8^{*}}{\text { Site-Gruppe }}$} \\
\hline & Site-Gruppe & Faktorgruppe & Site-Gruppe & Faktorgruppe & Site-Gruppe & Faktorgruppe & \\
\hline $\mathrm{T}_{\mathrm{d}}$ & $\mathrm{S}_{4}$ & $\mathrm{C}_{4 \mathrm{~h}}$ & $\mathrm{C}_{\mathrm{s}}$ & $\mathrm{D}_{2 \mathrm{~h}}$ & $\mathrm{C}_{1}$ & $\mathrm{C}_{2 \mathrm{~h}}$ & $\mathrm{C}_{2}$ \\
\hline $\mathrm{A}_{1}$ & $\mathrm{~A}$ & $A_{g}, B_{u}$ & $\mathbf{A}^{\prime}$ & $\begin{array}{c}A_{1 \mathrm{~g}}, \mathrm{~B}_{2 \mathrm{~g}}, \\
\mathbf{B}_{1 \mathrm{u}}, \mathbf{B}_{3 \mathrm{u}}\end{array}$ & $\mathbf{A}$ & $\begin{array}{c}\mathrm{A}_{\mathrm{g}}, \mathbf{A}_{\mathrm{u}}, \\
\mathrm{B}_{\mathrm{g}}, \mathbf{B}_{\mathrm{u}}\end{array}$ & $\mathbf{A}$ \\
\hline $\mathrm{E}$ & $A, \mathbf{B}$ & $\begin{array}{c}\mathrm{Ag}_{\mathrm{g}}, \mathrm{B}_{\mathrm{u}}, \\
\mathrm{B}_{\mathrm{g}}, \mathrm{A}_{\mathrm{u}}\end{array}$ & $\mathbf{A}^{\prime}, \mathbf{A}^{\prime \prime}$ & $\begin{array}{c}A_{1 \mathrm{~g}}, \mathrm{~B}_{2 \mathrm{~g}} \\
\mathbf{B}_{1 \mathrm{u}}, \mathbf{B}_{3 \mathrm{u}} \\
\mathrm{A}_{\mathrm{u}}, \mathrm{B}_{1 \mathrm{~g}} \\
\mathrm{~B}_{3 \mathrm{~g}}, \mathbf{B}_{2 \mathrm{u}}\end{array}$ & $2 \mathrm{~A}$ & $\begin{array}{c}2 A_{\mathrm{g}}, \mathbf{2} \mathbf{A}_{\mathrm{u}} \\
2 \mathrm{~B}_{\mathrm{g}}, \mathbf{2} \mathrm{B}_{\mathrm{u}}\end{array}$ & $2 \mathrm{~A}$ \\
\hline $\mathrm{F}_{2}$ & $\mathbf{B}, \mathbf{E}$ & $\begin{array}{l}\mathrm{B}_{\mathrm{g}}, \mathbf{A}_{\mathrm{u}}, \\
\mathrm{E}_{\mathrm{g}}, \mathbf{E}_{\mathrm{u}}\end{array}$ & $\mathbf{2} \mathbf{A}^{\prime}, \mathbf{A}^{\prime \prime}$ & $\begin{array}{c}2 \mathrm{~A}_{1 \mathrm{~g}}, 2 \mathrm{~B}_{2 \mathrm{~g}} \\
\mathbf{2} \mathbf{B}_{1 \mathrm{u}}, \mathbf{2} \mathbf{B}_{3 \mathrm{u}} \\
\mathrm{A}_{\mathrm{u}}, \mathrm{B}_{1 \mathrm{~g}} \\
\mathrm{~B}_{3 \mathrm{~g}}, \mathbf{B}_{2 \mathrm{u}}\end{array}$ & $\mathbf{3 A}$ & $\begin{array}{c}3 \mathrm{~A}_{\mathrm{g}}, \mathbf{3} \mathbf{A}_{\mathrm{u}} \\
3 \mathrm{~B}_{\mathrm{g}}, \mathbf{3} \mathbf{B}_{\mathrm{u}}\end{array}$ & $\mathbf{A}+\mathbf{2} \mathbf{B}$ \\
\hline
\end{tabular}

Tab. 2. Beziehungen zwischen den irreduziblen Darstellungen der Punktgruppe des freien Ions, der Site-Gruppe und der Faktorgruppe und die sich daraus ergebenden Auswahlregeln (IR-aktive Schwingungen halbfett). $\left.{ }^{*} \mathrm{Für} \mathrm{Ba}_{(\mathrm{MnO}}\right)_{2}$ wurde eine Faktorgruppenanalyse nicht durchgeführt, da die Anordnung der Ionen in der Elementarzelle nicht eindeutig bekannt ist ${ }^{23}$. 


\begin{tabular}{|c|c|c|c|c|c|c|}
\hline & $\begin{array}{l}\text { Raum- } \\
\text { gruppe }\end{array}$ & Lit. & $v_{1}$ & $\nu_{2}$ & $v_{3}$ & $v_{4}$ \\
\hline $\begin{array}{l}\mathrm{KMnO}_{4} \\
\mathrm{NH}_{4} \mathrm{MnO}_{4} \\
\mathrm{RbMnO}_{4} \\
\mathrm{CsMnO}_{4} \\
\mathrm{AgMnO}_{4} \\
\mathrm{Ba}\left(\mathrm{MnO}_{4}\right)_{2}\end{array}$ & $\begin{array}{l}D_{2 h}^{16} \\
D_{2 h}^{16} \\
D_{2 h}^{16} \\
D_{2 h}^{16} \\
C_{2 h}^{5} \\
D_{2 h}^{24}\end{array}$ & $\begin{array}{l}20 \\
21 \\
21 \\
21 \\
22 \\
23\end{array}$ & $\begin{array}{l}845(\mathrm{~s}) \\
845(\mathrm{~s}) \\
845(\mathrm{~s}) \\
845(\mathrm{~s}) \\
800(\mathrm{ss}) ? \\
843(\mathrm{~m})\end{array}$ & $\begin{array}{c}? \\
? \\
? \\
? \\
* \\
350(\mathrm{~s}) ?\end{array}$ & $\begin{array}{l}930 \text { (st), } 907 \text { (st) } \\
923(\text { st }) 903(\text { st }) \\
920(\text { Sch }), 910(\mathrm{Sch}), 900(\mathrm{st}) \\
920(\mathrm{Sch}), 910(\mathrm{Sch}), 900(\mathrm{st}) \\
898(\mathrm{~m}), 882(\mathrm{Sch}), 852(\mathrm{~m}) \\
940(\mathrm{st}), 930(\mathrm{Sch}), 920(\mathrm{st}), 880(\mathrm{st})\end{array}$ & $\begin{array}{l}400(\mathrm{~m}), 385(\mathrm{~m}), 381(\mathrm{Sch}) \\
400(\mathrm{~m}), 387(\mathrm{~m}), 382(\mathrm{Sch}) \\
400(\mathrm{~m}), 387(\mathrm{~m}), 382(\mathrm{Sch}) \\
400(\mathrm{~m}), 390(\mathrm{~m}), 383(\mathrm{~m}) \\
* \\
398(\mathrm{~m}), 378(\mathrm{~m}), 364(\mathrm{~s}) ?\end{array}$ \\
\hline $\begin{array}{l}\mathrm{NH}_{4} \mathrm{TcO}_{4} \\
\mathrm{CsTcO}_{4} \\
\mathrm{TlTcO}_{4} \\
\mathrm{AgTcO}_{4}\end{array}$ & $\begin{array}{l}\mathrm{C}_{4 \mathrm{~h}}^{6} \\
\mathrm{D}_{2 \mathrm{~h}}^{16} \\
\mathrm{D}_{2 \mathrm{~h}}^{16} \\
\mathrm{C}_{4 \mathrm{~h}}^{6}\end{array}$ & $\begin{array}{l}24 \\
24 \\
24 \\
24\end{array}$ & $\begin{array}{l}- \\
? \\
? \\
-\end{array}$ & $\begin{array}{l}348(\mathrm{~m}) \\
347(\mathrm{~s}) \\
347(\mathrm{~m}) \\
\quad *\end{array}$ & $\begin{array}{l}925(\text { Sch }), 900 \text { (st) } \\
920 \text { (Sch), } 910 \text { (st), } 895(\text { Sch }) \\
900 \text { (Sch), } 875 \text { (st), } 860 \text { (Sch) ? } \\
900 \text { (st), } 865 \text { (st) }\end{array}$ & $\begin{array}{l}329(\mathrm{st}), 317 \text { (st) } \\
330(\mathrm{~m}), 325(\mathrm{~m}) \\
332(\mathrm{st}), 327(\mathrm{st}), 322(\mathrm{st}) \\
*\end{array}$ \\
\hline $\begin{array}{l}\mathrm{KReO}_{4} \\
\mathrm{NH}_{4} \mathrm{ReO}_{4} \\
\mathrm{RbReO}_{4} \\
\mathrm{CsReO}_{4} \\
\mathrm{AgReO}_{4} \\
\mathrm{TlReO}_{4} \\
\mathrm{NaReO}_{4} * *\end{array}$ & $\begin{array}{l}\mathrm{C}_{4 h}^{6} \\
\mathrm{C}_{4 h}^{6} \\
\mathrm{C}_{4 h}^{6} \\
\mathrm{D}_{2 \mathrm{~h}}^{16} \\
\mathrm{C}_{4 \mathrm{~h}}^{6} \\
\mathrm{D}_{2 \mathrm{~h}}^{16} \\
\mathrm{C}_{4 \mathrm{~h}}^{6}\end{array}$ & $\begin{array}{l}25 \\
25 \\
25 \\
25 \\
26 \\
25 \\
25\end{array}$ & $\begin{array}{c}- \\
- \\
\bar{?} \\
\overline{-} \\
965(\mathrm{ss}) ? \\
868(6)\end{array}$ & $\begin{array}{l}361(\mathrm{~m}) \\
361(\mathrm{~m}) \\
350(\mathrm{~m}) \\
335(\mathrm{~m}) \\
315(\mathrm{ss}) ? \\
347(\mathrm{~m}) \\
360(1)\end{array}$ & $\begin{array}{l}929(\mathrm{Sch}), 910(=915-898) * *(\mathrm{st}) \\
930(\mathrm{Sch}), 910(=914-904) * *(\mathrm{st}) \\
935(\mathrm{Sch}), 915(\mathrm{st}) \\
935(\mathrm{Sch}), 920(\mathrm{st}), 907(\mathrm{Sch}) \\
910(\mathrm{Sch}), 880(\mathrm{st}) \\
920(\mathrm{Sch}), 900(\mathrm{st}), 890(\mathrm{st}) \\
928(2), 888(3)\end{array}$ & $\begin{array}{l}317 \text { (st), } 304(\mathrm{st}) \\
318 \text { (st, } 303 \text { (st) } \\
316 \text { (st), 308(st) } \\
320 \text { (st), 315(st) } \\
296 \text { (st), } 278 \text { (st) } \\
307 \text { (st) } \\
334(1)\end{array}$ \\
\hline
\end{tabular}

Tab. 3. IR-Spektren (innere Schwingungen der $\mathrm{MeO}_{4} \ominus$-Ionen; vgl. Text) wasserfreier Permanganate, Pertechnetate und Perrhenate (Frequenzen in $\mathrm{cm}^{-1}$ ). (st) : stark, (m) : mittel, (s) : schwach, (ss) : sehr schwach, (Sch) : Schulter. Bei vielen Banden, bei denen sich eine Aufspaltung nur schwach als Schulter andeutet, kann es sich auch um Kombinationsschwingungen mit Gitterschwingungen handelt. * Nicht gemessen. ** Die von und in einer früheren Arbeit ${ }^{15}$ und von Busey und KeLler ${ }^{4 \mathrm{c}}$ angegebene Aufspaltung der Bande mit einem Zentrum bei $910 \mathrm{~cm}^{-1}$ ist nur schwach zu erkennen. ${ }^{* * *}$ Von Fonteyne ${ }^{13}$ gemessene R a m a - Frequenzen (hier richtig zugeordnet).

genüber der nach der Site-Symmetrie $\mathrm{S}_{4}$ zu erwartenden führt ${ }^{1}$. Dies rechtfertigt eine Zuordnung der inneren Schwingungen der $\mathrm{MeO}_{4}{ }^{\ominus}$-Ionen in den hier untersuchten Permanganaten, Pertechnetaten und Perrhenaten nach der Site-Gruppe. Eine entsprechende Beobachtung, daß nämlich Korrelationsfeld-Aufspaltungen selten beobachtet werden, haben STEGER und DANZER $^{8}$ an zahlreichen kristallinen, wasserfreien Sulfaten und Phosphaten gemacht.

Auch die Auswahlregeln der Site-Symmetrie werden bei einigen der Salze nur andeutungsweise befolgt. Oft ist nicht eindeutig zu sagen, ob es sich bei den in den Spektren angedeuteten Schultern um Faktorgruppen- bzw. Site-Gruppen-Grundschwingungen handelt oder um Kombinationsschwingungen mit Gitterschwingungen. Eine Zuordnung dieser Art Kombinationsschwingungen ist sehr problematisch, wie Mrtra ${ }^{27}$ kürzlich gezeigt hat, da in Gitter-Kombinationsschwingungen auch $K \neq \mathrm{O}$-Phononen auf-

20 Strukturbericht I, 344; II, 415; S. Ramaseshan, K. Venkatesan u. N. V. Mani, Proc. Indian Acad. Sci. Sect. A 46, 95 [1957].

21 A. Müller u. B. Krebs, Naturwissenschaften 52, 494 [1965].

22 K. Sasvari, Z. Kristallogr., Mineralog. Petrogr., Abt. A 99, 9 [1938].

23 A. Hardy, C. Piekarski u. P. Hagen-Muller, C. R. hebd. Séances Acad. Sci. 249, 2579 [1959]. treten können. Wie schon von Steger und Danzer ${ }^{8}$ beobachtet worden ist, läßt sich besonders bei Alkaliverbindungen mit geringer polarisierender Wirkung des Kations eine nach den Auswahlregeln der SiteGruppe zu erwartende Aufspaltung der entarteten Schwingungen ( $\mathrm{E}$ und $\mathrm{F}_{2}$ des freien Ions) nicht beobachten. Bei fast allen untersuchten Verbindungen, die in der Raumgruppe $\mathrm{D}_{2 \mathrm{~h}}^{16}$ und $\mathrm{C}_{2 \mathrm{~h}}^{5}$ kristallisieren, ist eine dreifache deutliche Aufspaltung der $v_{3}\left(\mathrm{~F}_{2}\right)$. Bande nicht zu erkennen. Entsprechendes gilt für die $v_{4}\left(\mathrm{~F}_{2}\right)$-Schwingung. Bei den in der Raumgruppe $\mathrm{C}_{4 \mathrm{~h}}^{6}$ kristallisierten Pertechnetaten und Perrhenaten werden dagegen die Auswahlregeln der Site-Gruppe befolgt.

Eine interessante Ausnahme gegenüber den Spektren der anderen Salze bildet das Spektrum des Bariumpermanganats. Hier spaltet $v_{3}\left(\mathrm{~F}_{2}\right)$ deutlich dreifach auf. Die weiterhin auftretende Schulter bei $930 \mathrm{~cm}^{-1}$ läßt eventuell auf zusätzliche Korrelations-

24 C. Keller u. B. Kanellakopulos, Radiochim. Acta 1, 107 [1963] : B. J. McDonald u. G. I. Tyson, Acta crystallogr. [London] 15, 87 [1962].

25 J. Berntema, Z. Kristallogr., Mineralog. Petrogr. Abt. A 97, 300 [1937].

26 F. Buschendorf, Z. physik. Chem. B 20, 237 [1933].

27 S. S. Mitra, J. chem. Physics 39, 3031 [1963]. 
feld-Aufspaltung schließen. Ebenso ist die Bande bei $350(\mathrm{~s}) \mathrm{cm}^{-1}$ auffällig, die in anderen Permanganaten nicht beobachtet wird. Nach den Auswahlregeln der Site-Symmetrie ist nämlich in allen hier untersuchten Permanganaten $v_{2}$ IR-aktiv, wobei sich jedoch in allen Spektren für diese Schwingung keine Anzeichen andeuten. Da die Abweichungen von $\mathrm{T}_{d}$ Symmetrie beim $\mathrm{Ba}\left(\mathrm{MnO}_{4}\right)_{2}$ nach dem IR-Spektrum am deutlichsten sind, ist zu vermuten, daß die Bande bei $350 \mathrm{~cm}^{-1} v_{2}$ zuzuordnen ist. Die spektroskopische Sonderstellung des $\mathrm{Ba}\left(\mathrm{MnO}_{4}\right)_{2}$ ist auf die stärker polarisierende Wirkung des $\mathrm{Ba}^{2}{ }^{\odot}$-Ions zurückzuführen.

Die Frequenzen der inneren Schwingungen variieren beim Vergleich der verschiedenen Permanganate, Pertechnetate und Perrhenate jeweils untereinander mit Ausnahme der Silberverbindungen (und in geringerem Maße der Tl-Salze) nur sehr wenig. Die Silbersalze dagegen zeigen bei allen Schwingungsfrequenzen eine Verschiebung nach längeren Wellenlängen. Als Erklärung hierfür ist der im Gegensatz zu den anderen Salzen stärkere kovalente Bindungsanteil der Kationen-Sauerstoff-Bindungen anzunehmen.

\section{Experimentelles}

Darstellung der untersuchten Verbindungen: Die Permanganate wurden durch Umsetzung von $\mathrm{Ba}\left(\mathrm{MnO}_{4}\right)_{2}$ mit äquivalenten Mengen der entsprechenden Sulfate

28 W. Muthmann, Ber. dtsch. chem. Ges. 26, 1017 [1893]; vgl. G. Brauer, Handbuch der präparativen anorganischen Chemie, Band II, Enke-Verlag, Stuttgart 1951. in wäßriger Lösung dargestellt. Die filtrierten Lösungen wurden i. V. eingedampft, die erhaltenen Salze wurden einige Male umkristallisiert. Das Bariumpermanganat wurde nach Muthmann ${ }^{28}$ aus $\mathrm{KMnO}_{4}$ über $\mathrm{BaMnO}_{4}$ und anschließende Disproportionierung durch Einleiten von $\mathrm{CO}_{2}$ dargestellt. $\mathrm{CsTcO}_{4}$ und $\mathrm{AgTcO}_{4}$ wurden aus Lösungen von $\mathrm{NH}_{4} \mathrm{TcO}_{4}{ }^{29}$ durch Zugabe der Sulfate als recht schwerlösliche Niederschläge ausgefällt; ebenso wurden die Perrhenate mit $\mathrm{Me}=\mathrm{Rb}$, Cs, Ag und $\mathrm{Tl}$ durch Umsetzung von $\mathrm{NH}_{4} \mathrm{ReO}_{4}$ (Fluka puriss.) oder $\mathrm{KReO}_{4}$ (Fluka puriss.) mit den Sulfaten dargestellt. Alle Salze wurden, wenn möglich, mehrere Male umkristallisiert (vgl. auch l. c. ${ }^{30}$ ).

Aufnahme der Spektren: Die IR-Spektren wurden mit einem Leitz-IR-Spektrographen im Bereich zwischen 4000 und $300 \mathrm{~cm}^{-1}$ sowie mit einem BeckmanIR 11-Gerät im Bereich unterhalb $300 \mathrm{~cm}^{-1}$ gemessen. Es wurden bei allen untersuchten Verbindungen NujolSuspensionen verwendet. Im Falle der Ag- und TlSalze sowie der meisten Permanganate war die Verwendung von $\mathrm{KBr}$ - und $\mathrm{CsBr}$-Küvettenfenstern schwierig, da die Komponenten mehr oder weniger schnell miteinander reagierten (zum Teil mußte daher eine dünne Polyäthylenfolie als Zwischenschicht benutzt werden).

Wir danken Herrn Prof. Dr. O. Glemser sehr für sein Interesse und für großzügige Unterstützung. Herrn Prof. Dr. J. Goubeau und Herrn Priv.-Doz. Dr. K. Dehnicke gilt unser Dank für die Gelegenheit zur Aufnahme eines Teils der Spektren. Weiterhin danken wir Frl. H. Schmelz für ihre Hilfe bei der Reindarstellung der untersuchten Salze. Der De u t s c h en F or -

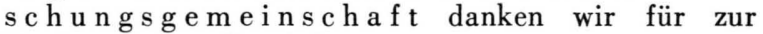
Verfügung gestellte Arbeitsgeräte.

$29 \mathrm{NH}_{4} \mathrm{TcO}_{4}\left({ }^{99} \mathrm{Tc}\right)$ der $\mathrm{Fa}$. Buchler, Braunschweig, mit weniger als $0,4 \%$ Re.

30 S. Tribalat, Rhénium et Technétium, Gauthier-Villars, Paris 1957. 